\title{
Aspectos relacionados à utilização de antirretrovirais em pacientes de alta complexidade no estado do Rio de Janeiro, Brasil
}

\author{
Aspects related to the use of antiretrovirals in high complexity \\ patients in the state of Rio de Janeiro
}

\author{
Lívia Gonçalves dos Santos Lima Madruga ${ }^{1}$ \\ Gabriela Veloso Vieira da Silva ${ }^{2}$ \\ Vinicius Abib Ramos Alves ${ }^{3}$ \\ Luis Guillermo Coca Velarde ${ }^{4}$ \\ Thiago Botelho Azeredo ${ }^{2}$ \\ Sergio Setúbal ${ }^{5}$ \\ Monique Araújo de Brito ${ }^{1}$ \\ Elisangela da Costa Lima ${ }^{2}$
}

\footnotetext{
${ }^{1}$ Programa de Pós-

Graduação em Ciências

Aplicadas a Produtos Para

a Saúde, Faculdade de

Farmácia, Universidade

Federal Fluminense (UFF).

R. Mário Viana 523, Santa

Rosa. 24241-000 Niterói RJ

Brasil. liviagdossantoslima@

gmail.com

${ }^{2}$ Observatório de Vigilância

e Uso de Medicamentos,

Faculdade de Farmácia,

Universidade Federal do Rio

de Janeiro. Rio de Janeiro

RJ Brasil.

${ }^{3}$ Curso de Graduação em

Farmácia, Faculdade de

Farmácia, UFF. Niterói RJ

Brasil.

${ }^{4}$ Instituto de Matemática e

Estatística, UFF. Niterói R

Brasil.

${ }^{5}$ Departamento de

Medicina Clínica/Serviço

de Doenças Infecciosas

e Parasitárias, Hospital

Universitário Antônio

Pedro, UFF. Niterói RJ

Brasil.
}

\begin{abstract}
Treatment of AIDS involves the use of the cocktail of drugs that make up the antiretroviral therapy. Its logistic control is monitored by a computerized national system of dispensation, the Logistic Control System of Medication (SICLOM). This study aimed to investigate, by means of SICLOM data, the use of antiretroviral therapy in patients treated at two University Hospitals in the state of Rio de Janeiro. A cross-sectional study was conducted with sociodemographic and dispensation data collected from SICLOM. The evaluation of drug ownership was done by calculating the Proportion of Days Covered (PDC). Five hundred and thirty-eight patients of both genders with active registration in SICLOM and over 18 years of age were included. The ART most used in both hospitals was lamivudine, considering the total of 58 different schemes identified. The mean of possession of groups was $88 \%( \pm 0,16)$. The factor associated with possession of drugs was the ART scheme, with PDC of $91 \%(p<0,001)$ for rescue schemes. This study confirmed that SICLOM was a reliable source to establish the profile of the population assisted.
\end{abstract}

Key words Antiretrovirals, Acquired Immunodeficiency Syndrome, Patient compliance
Resumo O tratamento da aids implica a utilização de diversos medicamentos que compõem a terapia antirretroviral, sendo o controle logístico monitorado por um sistema nacional informatizado de dispensação, o Sistema de Controle Logístico de Medicamentos (SICLOM). O objetivo foi investigar a utilização da terapia antirretroviral de pacientes de dois hospitais universitários no Estado do Rio de Janeiro a partir do SICLOM. Foi realizado um estudo seccional com coleta de dados sociodemográficos e de dispensação, a partir do SICLOM. A avaliação da posse para estimativa de pacientes não aderidos foi realizada através do indicador de proporção de dias cobertos (PDC). Foram incluídos 538 pacientes com cadastro ativo no SICLOM, maiores de 18 anos de ambos os sexos. $O A R V$ mais utilizado em ambos os hospitais foi lamivudina, dentre 58 esquemas diferentes identificados. A maior parte dos pacientes utilizava esquemas classificados como de primeira linha de tratamento. Em média, a proporção de dias cobertos (posse) pelo tratamento foi $88 \%( \pm 0,16)$. A linha de tratamento mostrou associação estatística com a posse, sendo observado melhor PDC 91\% ( $p<0,001)$ para os esquemas resgate. Este estudo corroborou o SICLOM como uma boa fonte para conhecimento do perfil de utilização de ARV.

Palavras-chave Antirretrovirais, Síndrome da Imunodeficiência Adquirida, Adesão à Medicação 


\section{Introdução}

Apesar dos avanços no tratamento, a pandemia da AIDS ainda é um problema de saúde pública. No ano de 2012, mais de 300 mil pessoas estavam em uso da terapia antirretroviral (ARV) no Brasil, de um total de 12 milhões em todo mundo ${ }^{1,2}$. O Rio de Janeiro foi o segundo Estado com maior número de óbitos por HIV/AIDS como causa básica² ${ }^{2}$. Os medicamentos ARV são distribuídos gratuitamente no Brasil desde $1996^{3}$ e o controle do estoque e do uso é feito por um sistema informatizado nacional, denominado Sistema de Controle Logístico de Medicamentos (SICLOM), que integra a Rede de Vigilância em AIDS ${ }^{4}$.

A era da terapia antirretroviral de alta eficiência contribuiu para o maior tempo de permanência no primeiro esquema antirretroviral, para um menor número de trocas de medicamentos, além de uma maior sobrevida das pessoas que vivem com HIV/AIDS (PVHA) ${ }^{5-7}$. No entanto, a detecção da utilização subótima dos medicamentos ainda é um importante cuidado para adequação do tratamento ${ }^{8}$.

De uma forma geral, os métodos mais utilizados para observação do comportamento dos pacientes quanto à da utilização de medicamentos são os indiretos, que não fornecem evidências objetivas de que o medicamento foi consumido, mas empregam estratégias substitutas, como entrevistas, questionários, contagem de comprimidos, dispositivos microeletrônicos e dados secundários (registros administrativos) ${ }^{8,9}$.

O uso de dados secundários é um recurso conveniente e custo-efetivo, especialmente, por conter informações de um grande número de pacientes ${ }^{8}$. No que tange a dispensação dos ARV, os registros oriundos de sistemas informatizados podem ser úteis para estimar o comportamento do paciente quanto à busca de medicamentos e, consequentemente, identificar os pacientes com baixa adesão e risco de falha virológica, devido a lacunas no período de tratamento preconizado ${ }^{9-16}$.

A adesão subótima aos ARV vem sendo observada em um relevante percentual de PVHA, de acordo com as pesquisas realizadas no Brasil $^{16-20}$. Estes estudos, contudo, ainda são escassos, apesar do monitoramento da farmacoterapia através do SICLOM estar expressamente indicado pelo Ministério da Saúde ${ }^{4,7}$. Este trabalho visou investigar aspectos relacionados ao uso dos ARV em dois hospitais universitários localizados no Estado do Rio de Janeiro, por meio de informações registradas no SICLOM.

\section{Metodologia}

Foi realizado um estudo exploratório seccional em farmácias cadastradas como unidades dispensadoras de medicamentos ARV, integradas à Secretaria Estadual de Saúde, em dois hospitais públicos de alta complexidade, localizados na região metropolitana do Estado do Rio de Janeiro ${ }^{21-23}$.

A amostra de estudo foi constituída por PVHA maiores de 18 anos, cadastradas entre 2008 e 2012 no Hospital A, e entre 1999 e 2014 no Hospital B. Em ambas as unidades, a seleção baseou-se na relação de cadastros ativos, obtida pelo aplicativo gerenciador de relatórios do SICLOM ${ }^{24}$. Dessa forma, não foram considerados os cadastros inativos, entendidos como os casos de abandono ou interrupção do tratamento, duplicidade de cadastro, óbitos, transferência para outra UDM, fim de gestação ou cadastro indevido ${ }^{25}$.

O critério de inclusão dos usuários foi definido de acordo a disponibilidade e confiabilidade na recuperação dos dados da unidade dispensadora de medicamentos (UDM), após a observação de documentos disponíveis em cada unidade. A definição deste critério considerou a conveniência na obtenção dos dados em ambos os hospitais $^{15,18,26}$, a saber: ter retiradas de medicamentos (data index) no intervalo de 01/01/2012 a 31/01/2012 no Hospital A e entre 01/11/2014 e 30/11/2014 no Hospital B. O período de inclusão foi determinante para a caracterização dos pacientes com cadastro ativo no SICLOM.

Em ambos os hospitais, foram excluídos os pacientes com apenas um registro de dispensação de ARV e gestantes ${ }^{16,26-28}$. Estas informações foram confirmadas a partir de consultas no prontuário físico e sistema de informação dos hospitais.

Informações acerca do sexo, idade, situação conjugal, escolaridade, raça, local de residência e tempo de tratamento foram coletadas no SICLOM. Ambas as unidades estavam realizando atualização de dados cadastrais dos pacientes durante o período de desenvolvimento da pesquisa. O procedimento de atualização do cadastro dos pacientes é uma demanda comum às UDM, que auxilia a busca ativa de usuários em atraso de dispensação. Para minimizar os dados faltantes (missings), foram consultados tanto o prontuário físico quanto o sistema informatizado de cada unidade.

O esquema ARV foi categorizado conforme o protocolo clínico e diretriz terapêutica (PCDT) vigente como (i) primeira linha de tratamento, (ii) segunda linha de tratamento, (iii) resgate e (iv) tratamento não recomendado no protocolo ${ }^{7,29}$. 
A análise da retirada e, portanto, da posse dos ARV considerou as informações registradas pelas farmácias sobre as múltiplas dispensações realizadas no período de estudo referentes (i) ao esquema ARV prescrito, (ii) à data da dispensação e (iii) à quantidade de ARV dispensada $a^{10,11,16-18,30-34}$.

Uma vez cadastrado por um serviço de farmácia, o usuário deve retirar seus ARV na mesma unidade. Como regra geral, as dispensações de ARV são realizadas para cobrir 30 dias de tratamento, podendo, em alguns casos, se estender para $60 \mathrm{ou}$, no máximo, 90 dias $^{7,35}$. O intervalo mínimo permitido entre duas retiradas de ARV é de 22 dias. Após um período de 90 dias sem retirada de ARV, o caso deve ser avaliado e o cadastro pode ser inativado ${ }^{7,35}$.

O método de registro de farmácia assume que os medicamentos resgatados anteriormente foram consumidos e que por isso há necessidade de nova retirada, sendo necessário mais de um registro de dispensação para que se possa estimar o consumo ${ }^{34,36,37}$. A literatura indica que o período de observação dos registros de dispensação pode ter início em uma data arbitrária ou ser determinado conforme o tratamento observado ${ }^{19,27,33,34}$. O período de coleta dos dados sobre a dispensação se estendeu por 180 dias, a partir da primeira dispensação registrada ${ }^{11,26,36,37}$.

A proporção de dias cobertos (PDC) foi escolhida como o indicador de posse dos medicamentos. A PDC é definida a partir do cálculo do número de dias em que o paciente dispunha de todos os medicamentos previstos no esquema ARV sobre o período total de observação (em dias), sem superposição ${ }^{19,27}$.

A data de dispensação foi registrada em todos os casos em que houve dispensação de, pelo menos, um ARV. A terapia ARV é composta por vários medicamentos sendo o esquema ideal composto de, pelo menos, três deles ${ }^{7}$. Nos casos em que não houve a retirada de algum dos ARV do esquema, a data de dispensação foi registrada, porém a posse de ARV foi considerada igual a zero ${ }^{27,31}$.

A PDC é apontada na literatura ${ }^{11,36,38}$ como uma estimativa da utilização subótima de medicamentos e neste estudo foi considerada como uma variável contínua, calculada a partir da média de posse de ARV da amostra do estudo.

A análise dos dados foi empreendida com auxílio do pacote estatístico R 3.2.2 e SPSS v.21. Foi realizado o teste $t$ de Student de comparação de médias no caso de variáveis numéricas e o teste de associação ANOVA para variáveis categóricas. Foram considerados os níveis de significância menores ou iguais a 0,05 e intervalos de confiança de $95 \%$.

A pesquisa foi aprovada nos Comitês de Ética em Pesquisa para os hospitais A e B.

\section{Resultados e discussão}

\section{Caracterização dos usuários e da terapia ARV dispensada}

Foram incluídos no estudo 538 usuários assistidos no hospital A (25\%) e B (75\%) (Figura 1) cuja caracterização está apresentada na Tabela 1.

A maior parte dos pacientes possuía entre 40 e 60 anos de idade, faixa etária esperada, considerando os avanços no tratamento ARV e aumento da sobrevida de PVHA ${ }^{5,6}$. Em média, os pacientes do hospital B eram 2,4 anos mais velhos do que os pacientes do hospital A. O perfil observado foi parecido ao de outros estudos nacionais ${ }^{18,19,39,40}$. Cerca de $60 \%$ dos usuários que compuseram a amostra eram do sexo masculino (Tabela 1), o que condiz com o atual perfil de $\mathrm{PVHA}^{2}$.

A amostra estudada possuía, em sua maior parte, alto grau de escolaridade, em ambos os hospitais. Alguns autores apontam que o baixo grau de instrução pode influenciar negativamente no tratamento ${ }^{41,42}$. A maioria dos usuários declarou-se parda, solteira e morador em uma cidade diferente do local de dispensação. Destaca-se o número de solteiros no Hospital $\mathrm{B}$, compondo cerca de $80 \%$ da amostra e nenhum casado. Alguns autores sugerem que o suporte social pode estar relacionado à forma pela qual o indivíduo conduz seu tratamento. Bonolo et al. ${ }^{20}$ demonstraram que mulheres casadas têm mais dificuldade em aderir ao tratamento quando comparado a homens casados. Kardas et al. apontaram que boa estrutura familiar favorece a adesão ao tratamento ${ }^{42}$. A informação sobre o estado civil é relevante para a equipe de saúde que acompanha PVHA, considerando-se a abordagem sobre prevenção da doença para os solteiros ${ }^{43}$ e a busca pelo suporte social em qualquer situação.

Foram encontrados 58 esquemas diferentes e a maioria da amostra recebia esquemas de primeira linha de tratamento (Tabela 1). As mudanças de critérios de início de tratamento aliadas às mudanças no tratamento ARV poderiam justificar tais achados ${ }^{5,6,44}$. Considerando as trocas de tratamento, foram prescritos 34 diferentes esquemas ARV no hospital A. Sete esquemas preconizados como primeira linha, 11 esquemas como segunda linha, 12 esquemas resgate e os outros 


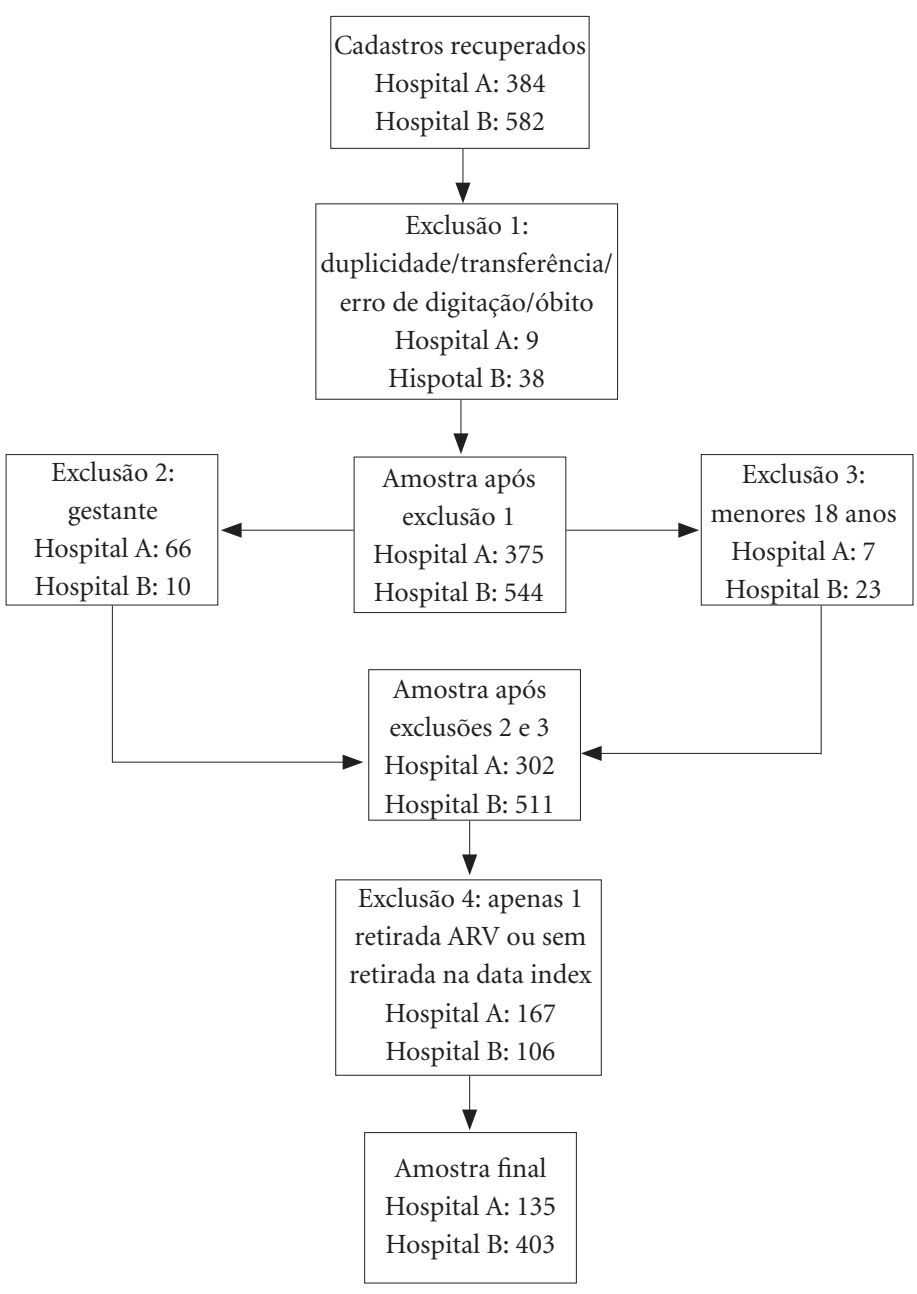

Figura 1. Número de pacientes incluídos e excluídos do banco de dados dos Hospitais A e B. Niterói, Rio de Janeiro, Brasil, 2015.

quatro não estavam previstos nas diretrizes de tratamento vigentes na época (Tabela 2). Neste hospital, 44\% (72) estavam em uso de esquemas

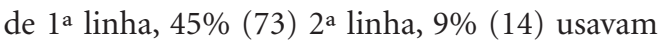
esquemas resgate, enquanto 3\% (5) usavam os considerados não classificados. No hospital B, foram encontrados 46 esquemas, dos quais cinco foram classificados como de primeira linha, 11 como de segunda linha, 15 como de resgate e 15 como não classificáveis segundo o protocolo clínico (Tabela 2). Aproximadamente, 47\% (199) da amostra do hospital B estavam em uso de esquemas de $1^{\text {a }}$ linha, 40\% (168) usaram esquemas

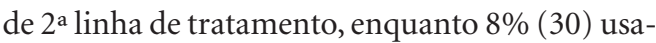
ram esquemas resgate, os outros 6\% (24) usaram esquemas não classificáveis. Ao longo do tempo, a adesão ao tratamento tende a diminuir ${ }^{5,6,41,45} \mathrm{e}$ uma das implicações da não adesão ao tratamento ARV é a resistência viral e falha virológica, que justificaria a troca de tratamento ${ }^{13}$ e o uso de esquemas resgate e não classificados pelo $\mathrm{PCDT}^{7}$. Por outro lado, avanços de conhecimento sobre os ARV permitiram que os pacientes permanecessem mais tempo em uso de esquemas de $1^{\text {a }}$ linha de tratamento ${ }^{5-7}$.

Considerando a complexidade do tratamento antirretroviral, eram esperadas diferentes combinações terapêuticas. Os hospitais estudados são Centros de Referência no tratamento de PVHA $^{22,23}$ e podem concentrar casos mais graves, o que justificaria a diversidade de esquemas. Pondera-se que, o número de esquemas utilizados foi bastante superior aos 28 esquemas observados na pesquisa realizada em outro hospital universitá- 
Tabela 1. Descrição da amostra nos Hospitais A e B. Niterói, Rio de Janeiro, Brasil, 2015.

\begin{tabular}{|c|c|c|c|c|}
\hline \multirow{2}{*}{ Variáveis (n) } & \multicolumn{3}{|c|}{$\mathrm{N}(\%)$} & \multirow{2}{*}{ Valor de $\mathrm{p}$} \\
\hline & Hospital A & Hospital B & Total & \\
\hline Sexo (538) & & & & $0,387^{\star}$ \\
\hline Feminino & $61(45,2)$ & $165(40,9)$ & $226(42)$ & \\
\hline Masculino & $74(54,8)$ & $238(59,1)$ & $312(58)$ & \\
\hline Idade (anos) (538) & & & & $0,041^{\star *}$ \\
\hline Média \pm DP & $44,9(11,9)$ & $47,3(11,5)$ & $46,7(11,6)$ & \\
\hline Mediana & 43,9 & 48,4 & 47,2 & \\
\hline Escolaridade (410) & & & & $0,541^{\star}$ \\
\hline Sem instrução e menos de 1 ano & 0 & $6(1,8)$ & $6(1,5)$ & \\
\hline 1 a 3 anos & $3(4,2)$ & $7(2,1)$ & $10(2,4)$ & \\
\hline 4 a 7 anos & $19(26,4)$ & $81(24)$ & $100(24,4)$ & \\
\hline 8 a 11 anos & $25(34,7)$ & $106(31,4)$ & $131(32)$ & \\
\hline 12 anos ou mais & $25(34,7)$ & $138(40,8)$ & $163(39,8)$ & \\
\hline Cor $(465)$ & & & & $0,093^{\star}$ \\
\hline Amarelo & $1(1,2)$ & $1(0,3)$ & $2(0,4)$ & \\
\hline Branco & $36(44,4)$ & $148(38, \%)$ & $184(39,6)$ & \\
\hline Pardo & $29(35,8)$ & $189(49,2)$ & $218(46,9)$ & \\
\hline Negro & $15(18,5)$ & $46(12)$ & $61(13,1)$ & \\
\hline Local de residência (538) & & & & $0,000^{*}$ \\
\hline Mesma cidade & $99(73)$ & $159(40)$ & $258(48)$ & \\
\hline Outra cidade & $36(27)$ & $244(60)$ & $280(52)$ & \\
\hline Estado Civil (350) & & & & $0,000^{*}$ \\
\hline Casado & $12(20,7)$ & 0 & $12(3,4)$ & \\
\hline Divorciado & $8(13,8)$ & $29(9,9)$ & $37(10,6)$ & \\
\hline Solteiro & $33(56,9)$ & $241(82,5)$ & $274(78,3)$ & \\
\hline Viúvo & $5(8,6)$ & $22(7,5)$ & $27(7,7)$ & \\
\hline Linha de tratamento (538) & & & & $0,497^{\star}$ \\
\hline Primeira Linha & $67(50)$ & $192(48)$ & $259(48)$ & \\
\hline Segunda Linha & $53(39)$ & $160(39)$ & $212(39)$ & \\
\hline Resgate & $12(9)$ & $30(7)$ & $42(8)$ & \\
\hline Não classificado & $3(2)$ & $21(6)$ & $24(5)$ & \\
\hline Tempo de tratamento (538) & & & & $0,000^{\star * *}$ \\
\hline Média +-DP & $3,2(1,2)$ & $10,4(5,2)$ & $8,6(5,5)$ & \\
\hline Mediana & 3,5 & 11,1 & 8,0 & \\
\hline Troca ARV (538) & & & & $0,000^{*}$ \\
\hline Sim & $22(16,3)$ & $19(4,7)$ & $41(7,6)$ & \\
\hline Não & $113(83,7)$ & $384(95,3)$ & $497(92,4)$ & \\
\hline
\end{tabular}

${ }^{\star}$ Qui quadrado; ${ }^{* *}$ t Student; ${ }^{* * *}$ ANOVA

rio brasileiro no estado de Minas Gerais, em um período maior de estudo ${ }^{19}$.

As atualizações das diretrizes terapêuticas para o tratamento de adultos com HIV/AIDS têm trazido avanços substanciais, como a possibilidade de início do tratamento independentemente da contagem linfocitária e substituição ou inclusão de ARV em diferentes linhas de tratamento. Apesar destas alterações, manteve-se a estrutura das opções terapêuticas, sendo a primeira linha composta por dois inibidores nu- cleosídeos/nucleotídeos da transcriptase reversa (INTR) associados a um inibidor não nucleosídeo da transcriptase reversa (INNTR), e a segunda linha composta por dois INTR associados a um inibidor de protease com reforço de ritonavir (IP/r). Nos esquemas de resgate é preconizado o uso de diversos ARV, definidos caso a caso, considerando-se as associações de ITRN, ITRNN (Etravirina), IP/r (darunavir/r), raltegravir ou maraviroque, dependendo da suscetibilidade viral verificada por exames de genotipagem, que 
Tabela 2. Distribuição dos esquemas terapêuticos usados por paciente em cada hospital segundo a linha de tratamento, considerando as trocas. Niterói, Rio de Janeiro, Brasil, 2015.

\begin{tabular}{|c|c|c|c|}
\hline \multirow{2}{*}{$\begin{array}{c}\text { Linha de } \\
\text { Tratamento }\end{array}$} & \multirow[b]{2}{*}{ Esquemas ARV } & \multicolumn{2}{|c|}{$\mathrm{N}(\%)$} \\
\hline & & $\begin{array}{c}\text { Hospital } \\
\text { A }\end{array}$ & $\begin{array}{c}\text { Hospital } \\
\text { B }\end{array}$ \\
\hline \multirow[t]{7}{*}{ 1a Linha } & Efavirenz - lamivudina - tenofovir & $35(21,3)$ & $44(10,4)$ \\
\hline & Efavirenz - zidovudina + lamivudina & $25(15,2)$ & $123(29)$ \\
\hline & Nevirapina - lamivudina - tenofovir & $4(2,4)$ & $1(0,2)$ \\
\hline & Lamivudina - nevirapina - tenofovir & $4(2,4)$ & 0 \\
\hline & Abacavir - efavirenz- lamivudina & $2(1,2)$ & $3(0,7)$ \\
\hline & Nevirapina - zidovudina + lamivudina & $1(0,6)$ & $28(6,6)$ \\
\hline & Estavudina - lamivudina - nevirapina & $1(0,6)$ & 0 \\
\hline \multirow[t]{16}{*}{ 2a Linha } & Atazanavir - ritonavir - tenofovir + lamivudina & $18(11)$ & $68(16)$ \\
\hline & Lopinavir + ritonavir - tenofovir + lamivudina & $12(7,3)$ & $19(4,5)$ \\
\hline & Atazanavir - ritonavir - zidovudina+ lamivudina & $10(6,1)$ & $33(7,8)$ \\
\hline & Lopinavir + ritonavir - zidovudina + lamivudina & $10(6,1)$ & $17(4)$ \\
\hline & Fosamprenavir - ritonavir - tenofovir + lamivudina* & $4(2,4)$ & $12(2,8)$ \\
\hline & Abacavir - atazanavir - lamivudina - ritonavir & $4(2,4)$ & $8(1,9)$ \\
\hline & Fosamprenavir - Ritonavir - zidovudina + lamivudina ${ }^{*}$ & $4(2,4)$ & $2(, 05)$ \\
\hline & Abacavir - lamivudina - lopinavir + ritonavir & $4(2,4)$ & $1(0,2)$ \\
\hline & Atazanavir-estavudina-lamivudina-ritonavir & $2(1,2)$ & 0 \\
\hline & Atazanavir - didanosina - lamivudina - ritonavir & $1(0,6)$ & $5(1,2)$ \\
\hline & Estavudina-lamivudina-lopinavir+ritonavir & $1(0,6)$ & 0 \\
\hline & Atazanavir-zidovudina+lamivudina & $1(0,6)$ & 0 \\
\hline & Atazanavir-lamivudina-tenofovir & $1(0,6)$ & 0 \\
\hline & Estavudina-fosamprenavir-lamivudina-ritonavir & $1(0,6)$ & 0 \\
\hline & Abacavir - fosamprenavir - lamivudina - ritonavir & 0 & $2(1,2)$ \\
\hline & Atazanavir-didanosina-lamivudina & 0 & $1(0,2)$ \\
\hline \multirow[t]{21}{*}{ Resgate } & Darunavir - raltegravir - ritonavir - tenofovir + lamivudina & $3(1,8)$ & $13(3,1)$ \\
\hline & Darunavir - raltegravir - ritonavir - zidovudina + lamivudina & $2(1,2)$ & $1(0,2)$ \\
\hline & Darunavir- lamivudina- raltegravir -ritonavir & $2(1,2)$ & 0 \\
\hline & Lamivudina-lopinavir+ritonavir-raltegravir -tenofovir & $2(1,2)$ & 0 \\
\hline & Darunavir - etravirina - raltegravir - ritonavir - tenofovir + lamivudina & $1(0,6)$ & 1 \\
\hline & Darunavir - etravirina - raltegravir-ritonavir & 0 & $3(0,7)$ \\
\hline & Darunavir - efavirenz - raltegravir - ritonavir - tenofovir + lamivudina & 0 & $2(0,5)$ \\
\hline & Darunavir - raltegravir - ritonavir & 0 & $4(0,9)$ \\
\hline & Darunavir-etravirina -lamivudina-raltegravir-ritonavir & $1(0,6)$ & 0 \\
\hline & Darunavir - raltegravir - ritonavir - tenofovir & $1(0,6)$ & 0 \\
\hline & Darunavir -ritonavir-tenofovir-zidovudina+lamivudina & $1(0,6)$ & 0 \\
\hline & Darunavir - lamivudina -ritonavir-tenofovir & $1(0,6)$ & 0 \\
\hline & Darunavir - didanosina - lamivudina - raltegravir - ritonavir & 0 & $1(0,2)$ \\
\hline & Darunavir - nevirapina - raltegravir - ritonavir - tenofovir + lamivudina & 0 & $1(0,2)$ \\
\hline & Darunavir - efavirenz - ritonavir - lamivudina - tenofovir & 0 & $1(0,2)$ \\
\hline & Lopinavir + ritonavir - raltegravir & 0 & $1(0,2)$ \\
\hline & Atazanavir - lamivudina- raltegravir - ritonavir & 0 & $1(0,2)$ \\
\hline & Raltegravir - tenofovir + lamivudina & 0 & $1(0,2)$ \\
\hline & Darunavir - raltegravir - ritonavir - tenofovir - zidovudina + lamivudina & 0 & $1(0,2)$ \\
\hline & atazanavir-lamivudina-raltegravir-ritonavir-tenofovir & 0 & $1(0,2)$ \\
\hline & Enfurvitida-lopinavir + ritonavir - zidovudina + lamivudina & 0 & $1(0,2)$ \\
\hline
\end{tabular}

são avaliados com o auxílio da Câmara Técnica de aids da Secretaria Estadual de Saúde ${ }^{7}$.
Em ambos os hospitais, os esquemas de primeira e segunda linha continham o ITRN lami- 
Tabela 2. Distribuição dos esquemas terapêuticos usados por paciente em cada hospital segundo a linha de tratamento, considerando as trocas. Niterói, Rio de Janeiro, Brasil, 2015.

\begin{tabular}{|c|c|c|c|}
\hline \multirow{2}{*}{$\begin{array}{c}\text { Linha de } \\
\text { Tratamento }\end{array}$} & \multirow[b]{2}{*}{ Esquemas ARV } & \multicolumn{2}{|c|}{$\mathbf{N}(\%)$} \\
\hline & & $\begin{array}{c}\text { Hospital } \\
\text { A }\end{array}$ & $\begin{array}{c}\text { Hospital } \\
\text { B }\end{array}$ \\
\hline \multirow[t]{14}{*}{ NC } & Lamivudina - ritonavir- tenofovir & $2(1,2)$ & 0 \\
\hline & Efavirenz - lamivudina - lopinavir + ritonavir - tenofovir & $1(0,6)$ & $5(1,2)$ \\
\hline & Efavirenz - lopinavir + ritonavir / zidovudina + lamivudina & $1(0,6)$ & 0 \\
\hline & Abacavir - atazanavir -lamivudina - lopinavir + ritonavir - ritonavir & $1(0,6)$ & 0 \\
\hline & Lopinavir + ritonavir - tenofovir - zidovudina + lamivudina & 0 & $8(1,9)$ \\
\hline & Lamivudina - lopinavir + ritonavir & 0 & $3(0,7)$ \\
\hline & Abacavir - efavirenz - lamivudina - lopinavir + ritonavir & 0 & $1(0,2)$ \\
\hline & Atazanavir - efavirenz - ritonavir - tenofovir + lamivudina & 0 & $1(0,2)$ \\
\hline & Atazanavir - didanosina - ritonavir - tenofovir + lamivudina & 0 & $1(0,2)$ \\
\hline & Nevirapina - tenofovir - zidovudina + lamivudina & 0 & $1(0,2)$ \\
\hline & Atazanavir - ritonavir - tenofovir & 0 & $1(0,2)$ \\
\hline & Efavirenz - lopinavir + ritonavir - tenofovir - zidovudina + lamivudina & 0 & $1(0,2)$ \\
\hline & Abacavir - atazanavir - ritonavir - tenofovir + lamivudina & 0 & $1(0,2)$ \\
\hline & Ritonavir - saquinavir - zidovudina + lamivudina & 0 & $1(0,2)$ \\
\hline
\end{tabular}

Legenda: $\mathrm{NC}=$ não classificado. ${ }^{\star}$ Esquema considerado como $2^{\text {a }}$ linha de acordo com o protocolo publicado em 2013,

anteriormente esquemas contendo fosamprenavir eram considerados resgate terapêutico (BRASIL, 2008; BRASIL 2013).

vudina. Este ITRN também foi o mais observado nos esquemas de resgate. A lamivudina tem boa resposta de supressão virológica e atividade residual, podendo ser usada mesmo em alguns casos de resistência ${ }^{7}$. Dois outros estudos brasileiros $^{18,19}$, ambos realizados no estado de Minas Gerais, observaram elevado número de esquemas contendo zidovudina+lamivudina. À época do estudo, a associação zidovudina+lamivudina era a opção preferencial de tratamento recomendada pelo $\mathrm{MS}^{29}$.

As diretrizes terapêuticas orientam o cuidado, norteando a equipe de saúde frente às decisões a respeito da assistência prestada. Alguns autores, no entanto, observaram que nem sempre as recomendações são seguidas, pois cada indivíduo responde de uma maneira ao tratamento proposto $^{46}$. Gomes et al. ${ }^{18}$, num estudo realizado em 2009, encontraram dez esquemas (de um total de 43 utilizados) considerados "incompletos" devido à falta de algum ARV ${ }^{25}$. Hirsch et al. ${ }^{47}$ também encontraram, em 2011, esquemas ARV "contraindicados" (10\% a 14\%) em farmácias sem atuação do profissional farmacêutico ${ }^{47}$. Em nosso estudo observou-se que $5 \%$ dos esquemas utilizados não estavam preconizados pelo MS. Este percentual foi considerado baixo, quando comparado aos estudos mencionados anteriormente $^{18,47}$. O estado do Rio de Janeiro apresenta- va o maior número de óbitos associados a Aids ${ }^{2}$ e esquemas menos complexos e preconizados podem viabilizar a manutenção em tratamentos de primeira linha por longos períodos ${ }^{7,44}$. Além disso, por tratar-se de serviços especializados, espera-se que a vigilância sobre os esquemas ARV seja maior ${ }^{35,48,49}$.

\section{Comportamento relacionado à retirada dos medicamentos}

A literatura apresenta diversos indicadores para a análise da posse de medicamentos. A escolha destas medidas deve basear-se na pergunta da pesquisa, na disponibilidade dos dados e nas características da doença estudada ${ }^{19,27,33,34,36,38}$.

A Proportion of Days Covered (PDC - proporção de dias cobertos) e o Medication Possession Ratio (MPR - taxa de posse de medicamentos) estão entre os indicadores mais utilizados ${ }^{8,18,26,37}$. Enquanto o numerador da PDC considera como posse a retirada de todos os medicamentos prescritos, o numerador do MPR é a média da posse de cada medicamento retirado no período observado $^{11,19,27,38}$. A vantagem de usar a PDC em polifarmácia deve-se ao fato de que este indicador fornece um resultado mais conservador, quando usado na avaliação da posse de medicamentos de uma mesma classe terapêutica ${ }^{26,27,31}$. A terapia 
ARV só é eficaz se todos os medicamentos que compõem o esquema são utilizados simultaneamente pelos pacientes ${ }^{5-7}$.

A média de PDC da amostra estudada foi igual a $88 \%( \pm 0,16)$. A posse de ARV estimada foi melhor que a observada nos estudos internacionais e nacionais exemplificados a seguir.

Salinas et al. ${ }^{13}$ avaliaram a persistência ao tratamento de primeira linha de tratamento ARV em pacientes peruanos, através do indicador MPR. A persistência calculada foi de $66 \%$ e, a cada incremento de $10 \%$ na MPR, observouse uma redução no risco de progressão para os esquemas da segunda linha de tratamento. No Peru, os esquemas de primeira linha e de segunda linha preconizam a mesma associação indicada pelas atuais diretrizes brasileiras ${ }^{13}$.

O estudo brasileiro que avaliou, por registro de farmácia, a adesão de pacientes virgens de tratamento em Minas Gerais, realizado por Gomes et al. ${ }^{18}$, verificou uma alta prevalência de abandono $(30,3 \%)$ e de retirada irregular $(57,9 \%)$. Um dos fatores associados foi a ausência de IP/r nos esquemas, diferentemente do observado em 2013 por Salinas et al. ${ }^{13}$.

Outra pesquisa brasileira, realizada por Fonseca et al. ${ }^{19}$ também em Minas Gerais, indicou que os esquemas com IP/r se associavam a um relevante percentual de retirada irregular e de abandono de tratamento ${ }^{19}$.

Não se observou, neste estudo, associação entre a PDC e as variáveis sexo, faixa etária, estado civil, raça, local de residência, troca do esquema terapêutico e hospital de origem (Tabela 3). Notou-se, no entanto, uma tendência à diminuição da PDC, conforme aumento do tempo de tratamento. Sabe-se que a aids é uma doença crônica e ainda relacionada a muitos estigmas e existe preocupação quanto ao tempo de tratamento ${ }^{5,6} \mathrm{e}$ a posse de $\mathrm{ARV}^{11,13,14}$. Os resultados desta pesquisa reforçam a necessidade de estratégias de orientação e sensibilização de PVHA com maior tempo de uso de ARV.

As informações sobre escolaridade, estado civil e raça foram, em nosso estudo, aquelas com maior dificuldade de recuperação (tabela 1). Este fato pode estar associado à falta de conhecimento sobre a importância destas informações na prestação da assistência por parte dos digitadores, à falta de treinamento no uso do sistema ${ }^{50-52}$ ou a algumas modificações do próprio SICLOM ${ }^{28,53}$.

Por outro lado, a coleta de dados secundários per si está sujeita a missings, devido à adaptação dos sistemas administrativos para a utilização com fins clínicos e assistenciais ${ }^{32,51}$. Em um estu- do realizado na cidade do Rio de Janeiro avaliando os fatores preditivos do abandono do tratamento contra o HIV/Aids mediante a obtenção de informações de prontuário, o percentual de missings foi de $25 \%{ }^{54}$. Também é possível que os usuários tenham se recusado a fornecer os dados.

Houve associação entre a PDC e a linha de tratamento prescrita (tabela 3). A maior média da PDC esteve associada a esquemas de resgate e a menor a esquemas ARV não classificados, segundo os critérios do $\mathrm{PDCT}^{7,29}$. Os três esquemas ARV com maior percentual de utilização foram os mesmos nas duas unidades investigadas ( $\mathrm{Ta}-$ bela 2).

Com o avanço dos ARV e os conhecimentos acerca desses medicamentos, os tratamentos foram modificados e as opções terapêuticas se tornaram de mais fácil manejo, tanto para os pacientes quanto para a equipe de saúde $\mathrm{d}^{5-7}$. Os dados deste estudo reforçam a expectativa de manutenção dos pacientes nas opções de primeira e segunda linha de tratamento ${ }^{5,6,19}$.

As atualizações dos protocolos de tratamento e a reorganização dos ARV dentro das diferentes indicações e opções terapêuticas, aliadas ao entendimento da equipe de saúde sobre a importância de seguir os protocolos e recomendações nacionais, poderiam justificar o fato de que $48 \%$ da amostra utilizava ARV da primeira linha de tratamento ${ }^{5-7,29}$. Esse aspecto é relevante, sobretudo, pelo fato de que $69 \%$ estavam em tratamento há, no mínimo, quatro anos.

No entanto, é preocupante a existência de pacientes em uso de esquemas resgate, especialmente os multiexperientes, em que a falta de adesão pode levar à perda da efetividade de uma classe, minimizando as opções futuras de tratamento, $\mathrm{o}$ que, por conseguinte, por ter má influência sobre o sucesso do combate à doença ${ }^{7,55}$.

Espera-se que a evolução dos tratamentos e dos conhecimentos sobre os ARV contribuam para que os usuários permaneçam na primeira linha de tratamento, dada a possibilidade de supressão viral e boa qualidade de vida ${ }^{5,6,56}$. A tendência a um menor valor de PDC, observada em usuários do esquema de segunda linha pode estar relacionada aos inibidores de protease. Essa classe de ARV provoca muitas reações adversas, associadas principalmente ao trato gastrointesti$\mathrm{nal}^{7,18,57}$.

O valor da PDC observado para usuários com os esquemas resgate foi melhor que o esperado, considerando a maior complexidade terapêutica destes esquemas e a possibilidade de não ter havido adesão prévia ao tratamento ${ }^{7}$. No entanto, 
Tabela 3. Análise da associação entre as variáveis independentes e a posse de ARV nos hospitais estudados. Niterói, Rio de Janeiro, Brasil, 2015.

\begin{tabular}{|c|c|c|c|}
\hline Variável & Média & Desvio Padrão & $\mathrm{p}$ \\
\hline Faixa etária & & & 0,16 \\
\hline $18-25$ anos & 0,86 & 0,18 & \\
\hline $25-35$ anos & 0,87 & 0,18 & \\
\hline $35-45$ anos & 0,88 & 0,18 & \\
\hline $45-60$ anos & 0,89 & 0,15 & \\
\hline 60 anos ou mais & 0,93 & 0,12 & \\
\hline Sexo & & & 0,19 \\
\hline Masculino & 0,89 & 0,16 & \\
\hline Feminino & 0,87 & 0,17 & \\
\hline Tempo de ARV & & & 0,10 \\
\hline menos 1 ano & 0,94 & 0,16 & \\
\hline $1-2$ anos & 0,92 & 0,17 & \\
\hline $2-3$ anos & 0,88 & 0,18 & \\
\hline $3-4$ anos & 0,84 & 0,18 & \\
\hline 4 anos ou mais & 0,89 & 0,15 & \\
\hline Raça & & & 0,17 \\
\hline Amarela & 0,70 & 0,18 & \\
\hline Branca & 0,89 & 0,16 & \\
\hline Parda & 0,89 & 0,14 & \\
\hline Preta & 0,86 & 0,18 & \\
\hline Estado Civil & & & 0,96 \\
\hline Casado & 0,88 & 0,26 & \\
\hline Divorciado & 0,89 & 0,16 & \\
\hline Solteiro & 0,89 & 0,15 & \\
\hline Viúvo & 0,88 & 0,15 & \\
\hline Escolaridade & & & 0,16 \\
\hline Nenhuma & 0,95 & 0,06 & \\
\hline 1 a 3 anos & 0,88 & 0,23 & \\
\hline 4 a 7 anos & 0,87 & 0,18 & \\
\hline 8 a 11 anos & 0,87 & 0,17 & \\
\hline 12 anos ou mais & 0,91 & 0,13 & \\
\hline Local de Residência & & & 0,73 \\
\hline Mesma Cidade & 0,88 & 0,16 & \\
\hline Outra Cidade & 0,89 & 0,16 & \\
\hline Opção Terapêutica & & & $<0,01$ \\
\hline Primeira linha & 0,90 & 0,14 & \\
\hline Segunda Linha & 0,87 & 0,16 & \\
\hline Resgate & 0,91 & 0,16 & \\
\hline Não classificado & 0,75 & 0,23 & \\
\hline Trocar de Esquema ARV & & & 0,12 \\
\hline Sim & 0,92 & 0,16 & \\
\hline Não & 0,88 & 0,16 & \\
\hline Local de Tratamento & & & 0,44 \\
\hline Hospital A & 0,87 & 0,19 & \\
\hline Hospital B & 0,89 & 0,15 & \\
\hline
\end{tabular}


o melhor comportamento quanto às retiradas regulares dos medicamentos pode ser também explicado pela vulnerabilidade em se adquirir infecções oportunistas e por internações sucessivas desses pacientes sob maior risco, fazendo com que as experiências negativas acabassem por incentivar a adesão ${ }^{55,57,58}$.

As menores PDC foram encontradas entre os esquemas não classificáveis de acordo com as recomendações do PDCT. Esse resultado pode refletir uma tentativa de manejo da doença em pacientes com poucas opções terapêuticas ou ainda a não adesão dos prescritores aos protocolos de tratamento ${ }^{46}$. Não obstante, em outros estudos também se observa a presença de esquemas não recomendados pelo $\mathrm{PCDT}^{18,47}$, o que reforça a importância da divulgação do PDCT e de suas atualizações. Este estudo não avaliou os dados de carga viral, pois não era um dado disponível no SICLOM. Rocha et al. ${ }^{17}$ sugeriram que as informações de carga viral e dados do SICLOM fossem combinadas para avaliação do perfil de não adesão de PVHA no país. Atualmente, as UDM estão sendo orientadas a incluir tais dados no SI$\mathrm{CLOM}^{59}$. Espera-se que esta nova informação tenha completitude no sistema e possa ser útil para a equipe monitorar clinicamente os usuários.

Apesar de não ser baixa, a posse média observada foi inferior ao ponto de corte de $95 \%$ indicado na literatura ${ }^{16-19,60,61}$. Este dado deve servir de alerta para as unidades de assistência a pacientes com HIV/Aids, principalmente em unidades do Rio de Janeiro, estado em que o alto índice de óbitos associados à doença reforça a preocupação com o incentivo ao uso correto da farmacotera$\mathrm{pia}^{2,48,55}$.

Alguns estudos consideram que há boa adesão quando se observa a posse dos medicamentos acima de $95 \%$ e que há utilização subótima quando o intervalo obtido está entre 80 e 95\% $\%^{18,14,60,62}$. Considerar o processo de utilização de medicamentos como um comportamento dicotômico (adesão versus não adesão) é uma decisão difícil, uma vez que pode variar entre 0 e $100 \%{ }^{8}$. Mesmo que o comportamento do paciente seja referido como dicotômico, a utilização de medicamentos raramente ocorre como um evento do tipo "tudo ou nada". Por este motivo, neste estudo, optouse por considerar a variável continua, sem limitá -la aos parâmetros indicados acima.

A análise dos registros de dispensação de medicamentos oferece evidências sobre o recebimento (e posse) do medicamento. Estudos que utilizam este indicador para análise da adesão ao tratamento presumem, portanto, que o me- dicamento foi consumido. Contudo, questões relacionadas à complexidade do tratamento número de medicamentos associados e a posologia de cada medicamento - bem como o estigma da doença podem influenciar o comportamento do paciente, face às diversas recomendações de um profissional de saúde, que não se limitam exclusivamente à retirada dos medicamentos. Também é possível que casais vivendo com HIV/ Aids compartilhem os medicamentos retirados, impactando o período de retirada esperado na análise.

Diversos termos - aceitação, adesão, concordância, fidelidade e persistência - são utilizados na literatura para se referir a esse comportamento. Um maior detalhamento sobre a taxonomia destes conceitos e sobre as medidas (indicadores) da utilização dos medicamentos pode ser obtido pela leitura dos trabalhos mais recentes publicados neste campo $^{8,10,16,26}$. Alguns autores associam a disponibilidade ou falta de medicamentos, avaliada por indicadores, ao risco de não adesão ao tratamento ${ }^{11-13,62}$. O uso de indicadores da posse de medicamentos é viável e considerando que a falta de adesão ao tratamento da aids é um problema de saúde pública, o uso de SICLOM para identificação de casos de utilização subótima é indicado, como também demonstrado por outros trabalhos nacionais ${ }^{16-18}$.

Cabe considerar como limitações do estudo, a realização de um estudo observacional com coleta de dados secundários, o que poderia interferir na completitude das informações requeridas para avaliar a posse e seus fatores associados ${ }^{16,32,63}$. Soma-se a isto o fato de o SICLOM não ter sido desenhado para avaliar a adesão ao tratamento da aids e existirem, por isso, dificuldades na sua utilização como meio de conhecer o perfil da população assistida nas $\mathrm{UDM}^{32,53}$. Pela impossibilidade de acesso às informações de usuários com cadastro inativo no sistema, entende-se que a composição de uma amostra com cadastros ativos pode ter superestimado as análises. Destaca-se que ocorre inativação do cadastro em casos de interrupção do tratamento ou baixíssima adesão de PVHA. O cuidado, nestes casos, requer diferentes estratégias da equipe multidisciplinar. Assim, sua inclusão no estudo tornaria a amostra muito heterogênea, comprometendo a análise. Por outro lado, a estratégia do estudo com o uso do PDC como indicador de avaliação de posse de ARV permitiu a discussão do uso desta metodologia para identificação de PVHA com cadastro ativo e problemas da retirada do tratamento, e, consequentemente, a utilização sub-ótima deste, 
o que poderá contribuir com a realização de novos estudos no país.

\section{Conclusão}

Ainda são escassos os estudos sobre a utilização de ARV no Brasil e espera-se que este trabalho mostre a viabilidade de sua realização utilizando os sistemas disponíveis de informação. A não adesão ao tratamento traz prejuízos consideráveis à saúde dos pacientes e ameaça o controle da doença. Qualquer esforço realizado no sentido de melhorar a qualidade de vida dos indivíduos deve ser incentivado.

Os dados apresentados sugerem que as unidades seguem as atualizações e as recomendações das diretrizes nacionais de tratamento da aids. $\mathrm{O}$ conhecimento do perfil dos indivíduos assistidos nas unidades de saúde norteia os esforços e po- líticas locais que contribuem para a melhoria da assistência. Os aspectos associados ao tratamento da aids, obtidos do SICLOM, são os mesmos destacados na literatura em estudos que avaliam a (não) adesão. Os dados apresentados reforçam os achados de que, apesar de ser um sistema administrativo, o SICLOM pode ser utilizado para a realização de atividades assistenciais, favorecendo o conhecimento dos esquemas ARV prescritos e utilizados nas unidades de dispensação. A reunião de informações relevantes ao acompanhamento de pacientes em um sistema de informação é útil para o monitoramento clínico de pacientes em uso de ARV pela equipe assistencial. $\mathrm{O}$ indicador escolhido mostrou-se adequado para avaliar a posse de ARV e permitiu observar que pacientes em uso de esquemas resgate apresentaram maior posse de $\mathrm{ARV}$, o que poderia sugerir melhor adesão dos pacientes nos centros de referência investigados.

\section{Colaboradores}

Todos os autores contribuíram para a concepção, estruturação, análise e interpretação dos dados, e desenvolveram ou realizaram a revisão crítica do artigo, com o objetivo de obter um conteúdo intelectual condizente com esta revista.

\section{Agradecimentos}

A CAPES, CNPq e FAPERJ pelo fomento e incentivo ao desenvolvimento desta pesquisa. 


\section{Referências}

1. Organização Mundial da Saúde (OMS). Nota Descritiva $n^{0}$ 360. [acessado 2014 Set 4]. Disponível em: http:// www.who.int./mediacentre/factsheet s/fs360/en

2. Brasil. Portal sobre AIDS, DST e Hepatites Virais. Boletim Epidemiológico 2013. [acessado 2014 Jun 11]. Disponível em: http://www.aids.gov.br/sites/default/files/ anexos/publicacao/2013/55559/_p_boletim_2013_internet_pdf_p_51315

3. Brasil. Lei 9.313, de 13 de novembro de 1996. Dispõe sobre a distribuição gratuita de medicamentos aos portadores do HIV e doentes de AIDS. Diário Oficial da União 1996; 14 nov.

4. Brasil. Portal sobre AIDS, doenças sexualmente transmissíveis e Hepatites Virais. [acessado 2014 Jun 11]. Disponível em: http://www.aids.gov.br/node/365

5. Slama L, Xiuhong Li MAS, Brown T, Jacobson LP, Pialoux G, Macatangay B, Bolan RK, Phair J, Palella Junior FJ. Increases in Duration of First Highly Active Antiretroviral Therapy Over Time (1996-2009) and Associated Factors in the Multicenter AIDS Cohort Study. J Acquir Immune Defic Syndr 2014; 65(1):57-64.

6. Rossi SMG, Maluf ECP, Carvalho DS, Ribeiro CEL, Battaglin CRP. Impacto da terapia antirretroviral conforme diferentes consensos de tratamento da Aids no Brasil. Rev Panam de Salud Publica 2012; 32(2):117123.

7. Brasil. Ministério da Saúde (MS). Protocolo Clínico para Manejo da Infeç̧ão pelo HIV em adultos. Brasília: MS; 2013.

8. McCaffrey DJ. Padrões de utilização dos medicamentos. In: Yang, Y, West-Strum, organizadores. Comprendendo a Farmacoepidemiologia. Porto Alegre: AMGH; 2014. p. 132-152.

9. Berg KM, Arnsten JH. Practical and Conceptual Challenges in Measuring Antiretroviral Adherence. J Acquir Immune Defic Syndr 2006; 43(Supl. 1):79-87.

10. Vrijens B, Geest S, Hughes DA, Przemyslaw K, Demonceau J, Ruppar T, Dobbels F, Fargher E, Morrison V, Lewek P, Matyjaszczyk M, Mshelia C, Clyne W, Aronson JK, Urquhart J. A new taxonomy for describing and defining adherence to medications. Br J Clin Pharmacol 2012; 73(5):691-705.

11. McMahon JH, Jordan MR, Kelley K, Bertagnolio S, Hong SY, Wanke CA, Lewin SR, Elliott JH. Pharmacy Adherence Measures to Assess Adherence to Antiretroviral Therapy: Review of the Literature and Implications for Treatment Monitoring. Clin Infec Dis 2011; 52(4):493-506.

12. Inciardi JF, Leeds AL. Assessing the Utility of a Community Pharmacy Refill Record as a Measure of Adherence and Viral Load Response in Patients Infected with Human Immunodeficiency Virus. Pharmacotherapy 2005; 25(6):790-796.

13. Salinas JL, Alave JL, Westfall AO, Paz J, Moran F, Carbajal-Gonzalez D, Callacondo D, Avalos O, Rodriguez M, Gotuzzo E, Echevarria J, Willig JH. Medication Possession Ratio Predicts Antiretroviral Regimens Persistence in Peru. PLOS ONE 2013; 8(10):e75429.
14. Sangeda RZ, Mosha F, Prosperi M, Aboud S, Vercauteren J, Camacho RJ, Lyamuya EF, Wijngaerden EV, Vandamme AM. Pharmacy refill adherence outperforms self-reported methods in predicting HIV therapy outcome in resource-limited settings. BMC Public Health 2014; 14:1035.

15. Obreli-Neto PR, Baldoni AO, Guidoni CM, Bergamini D, Hernandes KC, Luz RT, Silva FB, Silva RO, Pereira LRL, Cuman RKN. Métodos de avaliação de adesão à farmacoterapia. Rev Bras Farm 2012; 93(4):403-410.

16. Ernesto AS, Lemos RMBP, Huehara MI, Morcillo AM, Vilela MMS, Silva MTN. Usefulness of pharmacy dispensing records in the evaluation of adherence to antiretroviral therapy in Brazilian children and adolescents. Braz J Infec Dis 2012; 16(4):315-320.

17. Rocha GM, Machado CJ, Acurcio FA, Guimarães MDC. Monitoring adherence to antirretroviral treatment in Brazil: an urgent challenge. Cad Saude Puública 2011; 27(Supl. 1):67-78.

18. Gomes RRFM, Machado CJ, Acurcio FA, Guimarães MDC. Utilização dos Registros de Dispensação da Farmácia como Indicador da Não-adesão à Terapia Anti-retroviral em Indivíduos Infectados pelo HIV. Cad Saude Publica 2009; 25(3):495-506.

19. Fonseca LC, Martins FJ, Vieira RCPA, Pereira RMC, Ferreira AS, Raposo NRB. Evaluation of inadequated anti-retroviral therapy in patients with HIV/AIDS. Rev Soc Bras Med Trop 2012; 45(2):151-155.

20. Bonolo PF, Ceccato MGB, Rocha GM, Acurcio FA, Campos LN, Guimarães MDC. Gender differences in non-adherence among Brazilian patients initiating antiretroviral therapy. Clinics 2013; 68(5):612-620.

21. Brasil. Portal do Cadastro Nacional de Estabelecimento de Saúde. [acessado 2015 Nov 29]. Disponível em: http://www2.datasus.gov.br/DATASUS/index.php?area $=0204$

22. Brasil. Portal sobre AIDS, doenças sexualmente transmissíveis e Hepatites Virais. [acessado 2014 jun 24]. Disponível em: http://www.aids.gov.br/tipo_endereco/ servico-de-assistencia-especializada-em-hivaids

23. Brasil. Portal sobre AIDS, doenças sexualmente transmissiveis e Hepatites Virais. [acessado 2015 Nov 21]. Disponível em: http://www.aids.gov.br/sites/default/ files/anexos/legislacao/2013/52759/portaria_conjunta_no_1_pdf_49335.pdf

24. Brasil. Portal sobre AIDS, doenças sexualmente transmissiveis e Hepatites Virais. [acessado 2016 Ago 31] Disponível em: http://azt.aids.gov.br/documentos/siclom_operacional/entrando.pdf

25. Brasil. Portal sobre AIDS, doenças sexualmente transmissíveis e Hepatites Virais. [acessado 2016 ago 31]. Disponível em: http://azt.aids.gov.br/documentos/siclom_operacional/cadastramento2013.pdf

26. Hess LM, Raebel MA, Conner DA, Malone DC. Measurement of Adherence in Pharmacy Administrative Databases: A Proposal for Standard Definitions and Preferred Measures. Ann Pharmacother 2006; 40(78):1280-1288. 
27. Raebel MA, Schmittdiel J, Karter AJ, Konieczny JL, Steiner JF. Standardizing Terminology and Definitions of Medication Adherence and Persistence in Research employing Electronic Databases. Med Care 2013; 51(8):S11-S21.

28. Brasil. Portal sobre AIDS, doenças sexualmente transmissiveis e Hepatites Virais. [acessado 2014 Jun 11]. Disponível em: http://azt.aids.gov.br/documentos/Guia\%20 de\%20referencia\%20rapida\%20siclom.pdf

29. Brasil. Ministério da Saúde (MS). Recomendações para terapia anti-retroviral em adultos infectados pelo HIV: manual de bolso. Brasília: MS; 2008.

30. Arnet I, Abraham I, Messerli M, Hersberger KEA. A method for calculating adherence to polypharmacy from dispensing data records. Int J Clin Pharm 2014; 36:192-201.

31. International Society for Pharmacoeconomics and Outcomes Research (ISPOR). [acessado 2014 Fev 2]. Disponível em: https://www.ispor.org/student/teleconferences/ISPORStudentForumPresentation022410.pdf

32. Soares C, Silva GA. Uso de registros de assistência farmacêutica do Sistema de Informação Ambulatorial para avaliação longitudinal de utilização e adesão a medicamentos. Cad de Saúde Coletiva 2013; (21):245-252.

33. Kozma CM, Dickson M, Phillips AL, Meletiche DM. Medication possession ratio: implications of using fixed and variable observation periods in assessing adherence with disease-modifying drugs in patients with multiple sclerosis. Patient Prefer Adherence 2013; 12(7):509-116.

34. Steiner JF, Prochaka AV. The Assessment of Refill Compliance Using Pharmacy Records: Methods, Validity, and Applications. J Clinical Epimiology 1997; 50(1):105-116.

35. Brasil. Protocolo da Assistência Farmacêutica em DST/ HIV/Aids: recomendações do Grupo de Trabalho de Assistência Farmacêutica. Brasília: MS; 2010.

36. Peterson AM, Nau DP, Cramer JA, Benner J, Gwadry-Sridhar F, Nichol M. A checklist for medication compliance and persistence studies using retrospective databases. Value in Health 2007; 10(1):3-12.

37. Sattler ELP, Lee JS, Perri M. Medication (Re)fill Adherence Measures Derived from Pharmacy Claims Data in Older Americans: A Review of the Literature. Drugs \& Aging 2013; 30(6):383-399.

38. Andrade SE, Kahler KH, Frech F, Chan KA. Methods for evaluation of medication adherence and persistence using automated databases. Pharmacoepidemiol Drug Saf 2006; 15(8):565-574.

39. Romeu GA, Tavares MM, Carmo CP, Magalhães KN, Nobre ACL, Matos VC. Avaliação da Adesão a Terapia Antirretroviral de Pacientes portadores de HIV. Rev Bras Farm Hosp Serv Saúde 2012; 3(1):37-41.

40. Carvalho CV, Merchán-Hamann E, Matsushita R. Determinantes da adesão ao tratamento anti-retroviral em Brasília, DF: um estudo de caso-controle. Rev. Soc. Bras. Med. Trop. 2007; 40(5):555-565.
41. Brown MT, Bussell JK. Medication Adherence: WHO Cares? Mayo Clin Proc 2011; 86(4):304-314.

42. Kardas P, Lewek P, Matyjaszczyk M. Determinants of patients adherence: a review of systematic reviews. Front Pharmacol 2013, 4:91.

43. Alves MFP. Sexualidade e prevenção de DST/AIDS: representações sociais de homens rurais de um município da zona da mata pernambucana, Brasil. Cad Saude Publica 2003; 19(Supl. 2):S429-S39.

44. May M, Gompels M, Delpech V, Porter P, Post F, Johnson M, Dunn D, Palfreeman A, Gilson R, Gazzard B, Hill T, Walsh J, Fisher M, Orkin C, Ainsworth J, Bansi L, Phillips A, Leen C, Nelson M, Anderson J. Impact of late diagnosis and treatment on life expectancy in people with HIV-1: UK Collaborative HIV Cohort (UK CHIC) Study. British Medical Journal 2011; 343:d6016

45. Blaschke TF, Osterberg L, Vrijens B, Urquhart J. Adherence to medications: insights arising from studies on the unreliable link between prescribed and actual drug dosing histories. Annu Rev Pharmacol Toxicol 2012; 52:275-201.

46. Cabana MD, Rand CS, Powe NR, Wu AW, Wilson MH, Abboud PAC, Rubin HR. Why Don't Physicians Follow Clinical Practice Guidelines? JAMA 1999; 282(15):1458-1465.

47. Hirsch JD, Gonzales M, Rosenquist A, Miller TA, Gilmer TP, Best BM. Antiretroviral Therapy Adherence, Medication Use, and Health Care Costs During 3 Years of a Community Pharmacy Medication Therapy Management Program for Medi-Cal Beneficiaries with HIV/AIDS. J Manag Care Pharm 2011; 17(3):213-223.

48. Nemes MIB. Adesão ao Tratamento, acesso e qualidade da assistência em AIDS no Brasil. Rev. Assoc. Med. Bras. 2009; 55(2):207-212.

49. Brasil. Ministério da Saúde (MS). Avaliação da qualidade dos serviços ambulatoriais do SUS que assistem adultos vivendo com HIV/Aids no Brasil: Relatório da Aplicação de 2010 e Comparação com o resultado de 2007. São Paulo: MS; 2011.

50. Cruz MM, Toledo LM, Santos EM. O sistema de informação de AIDS do Município do Rio de Janeiro: suas limitações e potencialidades enquanto instrumento da vigilância epidemiológica. Cad Saude Publica 2003; 19(1):81-89.

51. Lima CRA, Schramm JMA, Coeli CM, Silva MEM. Revisão das dimensões de qualidade dos dados e métodos aplicados na avaliação dos sistemas de informação em saúde. Cad Saude Publica 2009; 25(10):2095-2109.

52. Freitas FP, Pinto IC. Percepção da equipe da saúde da família sobre a utilização do sistema te informação da atenção básica - SIAB. Rev Latino-am Enfermagem 2005; 13(4):547-554.

53. Sakita KM. Avaliação da implantação do Sistema de Controle Logístico de Medicamentos/Aids: O caso do Distrito Federal [dissertação]. Rio de Janeiro: Escola Nacional de Saúde Pública Sergio Arouca; 2012. 
54. Schilkowsky LB, Portela MC, Sá MC. Fatores associados ao abandono de acompanhamento ambulatorial em um serviço de assistência especializada em IV/aids na cidade do Rio de Janeiro, RJ. Rev Bras Epidemiol 2001; 14(2):187-197.

55. Brasil. Ministério da Saúde (MS). Manual de adesão ao tratamento para pessoas vivendo com HIV e aids. Manual de adesão ao tratamento para pessoas vivendo com HIV e Aids. Brasília: MS; 2008.

56. Barbosa CD, Balp MM, Kulich K, Germanin N, Rofai D. A literature review to explore the link between treatment satisfaction and adherence, compliance, and persistence. Patient Prefer Adherence 2012; 6:39-48.

57. Langebeek N, Gisolf EH, Resiss P, Vervoort SC, Hafsteinsdóttir TB, Richter C, Sprangers MAG, Nieuwkerk PT. Predictors and correlates of adherence to combination antiretroviral therapy (ART) for chronic HIV infection: a meta-analysis. BMC Medicine 2014, 12:142.

58. Bartlet JA, Hornberger J, Shewade A, Bhor M, Rajagopalan R. Obstacles and Proposes Solutions to Effective Antiretroviral Therapy in Resource - Limited Settings. J. Int. Assoc. Provid. AIDS Care 2009; 8(4):253-268.

59. Brasil. Portal sobre AIDS, doenças sexualmente transmissíveis e Hepatites Virais. [acessado 2016 Ago 30]. Disponível em: http://www.aids.gov.br/pagina/sistema-decontrole-logistico-de-medicamentos-siclom

60. Paterson DL, Swindells S, Mohr J, Brester M, Vergis EN, Squier C, Wagener MM, Singh N. Adherence to protease inhibitor therapy and outcomes in patients with HIV infection. Ann Intern Med 2000; 133(1):21-30.

61. Bonolo PF, Gomes RRFM, Guimaraes MDC. Adesão à terapia anti-retroviral (HIV/aids): fatores associados e medidas da adesão. Epidemiol Serv Saúde 2007; 16(4):261-278.

62. Gross R, Yip B, Lo Re V3rd, Wood E, Alexander CS, Harrigan PR, Bangberg DR, Montaner JS, Hogg RS. A Simple, dynamic measure of antiretroviral therapy adherence predicts failure to maintain HIV-1 suppression. J Infect Dis 2006; 194(8):1108-1114.

63. World Health Organization (WHO). Essential Medicines and Health Products Information Portal. [acessado 2015 Nov 29] Disponível em: http://apps.who.int/ medicinedocs/en/d/Js4876e/2.html

Artigo apresentado em 22/06/2016

Aprovado em 02/03/2017

Versão final apresentada em 04/03/2017 